\title{
Retro-mode imaging of fibrovascular membrane in proliferative diabetic retinopathy after intravitreal bevacizumab injection
}

This article was published in the following Dove Press journal:

Clinical Ophthalmology

29 June 2011

Number of times this article has been viewed

\author{
Kiyoshi Suzuma \\ Eiko Tsuiki \\ Makiko Matsumoto \\ Azusa Fujikawa \\ Takashi Kitaoka \\ Department of Ophthalmology \\ and Visual Sciences, Graduate School \\ of Biomedical Sciences, Nagasaki \\ University, Nagasaki, Japan
}

Correspondence: Kiyoshi Suzuma Department of Ophthalmology and Visual Sciences, Graduate School of Biomedical Sciences, Nagasaki University, Nagasaki 852-850I, Japan

Tel +8 I-95-8|9-73450। I

Fax +8I-95-8I 9-7347

Email suzuma@nagasaki-u.ac.jp
Abstract: The F10 is a new commercially available scanning laser confocal ophthalmoscope (SLO) that can perform multiple functions. We determined the usefulness of noninvasive evaluation of proliferative diabetic retinopathy (PDR) pathologies before and after intravitreal injection of bevacizumab (IVB) using the new indirect viewing system of the retro-mode function of the F10 SLO, and compared the images histologically with surgically excised fibrovascular membrane from two cases. In PDR, neovascular vessels in fibrovascular membrane were clearly seen with the retro-mode, even after IVB and without blood flow. The F10 SLO may be useful in evaluating neovascular vessels in fibrovascular membrane in PDR and for determining the precise retinal changes in diabetic retinopathy.

Keywords: bevacizumab, imaging, proliferative diabetic retinopathy, retro-mode, scanning laser ophthalmoscope

\section{Introduction}

Recent clinical trials of the intravitreal injection of bevacizumab (Avastin ${ }^{\circledR}$, Roche, Reinach, Switzerland) (IVB) have shown excellent results in the treatment of angiogenic pathologies including proliferative diabetic retinopathy (PDR). It has been reported that IVB significantly decreases bleeding from the vessels of the fibrovascular membrane during vitrectomy in PDR, and IVB has also been reported to be effective in the regression of new vessels in PDR. ${ }^{1-3}$

The F10 (Nidek, Gamagori, Japan) is a new commercially available scanning laser confocal ophthalmoscope (SLO) that can perform multiple functions including fluorescein angiography, indocyanine green angiography, fundus autofluorescence, retro-mode imaging, and dark-field mode imaging. In the dark-field mode with a central stop, scattered light from the deeper layers of the retina silhouettes structural abnormalities. In contrast, the retro-mode of the F10 SLO uses an infrared laser and an aperture with a modified central stop that is displaced laterally from the confocal light path. This optical arrangement allows for a clearer and pseudo-3-dimensional image, and is a new method of detecting abnormalities in the retina, such as cystoid macular edema ${ }^{4}$ or myopic retinoschisis. ${ }^{5}$ However, to our knowledge, no previous report has examined the fibrovascular membrane in PDR with the retro-mode.

Accordingly, we used the new indirect viewing system of the retro-mode function of the F10 SLO for noninvasive evaluation of PDR pathologies before and after IVB, and compared the images histologically with surgically excised fibrovascular membrane from two cases. 


\section{Cases and findings Case I (Figure I)}

A 52-year-old man with a history of PDR in the left eye was examined. Best corrected visual acuity (BCVA) in this eye was 20/300. Fundus examination disclosed very severe fibrovascular proliferation throughout the posterior pole (Figure 1A). Interestingly, in the retro-mode imaging with the F10 SLO, the neovascular vessels in the fibrovascular membrane were clearly observed, as in angiography. However, the outline of the fibrovascular membrane itself was not clear (Figure 1B). Three days after $1.25 \mathrm{mg}$ of IVB, fundus examination disclosed regression of most of the new vessels in the significantly contracted fibrovascular membrane, consistent with previous reports ${ }^{6,7}$ (Figure 1C). Surprisingly, most neovascular vessel structures in the fibrovascular membrane were still clearly observed in the retro-mode image (Figure 1D).

\section{Case 2 (Figure 2)}

A 39-year-old man with a history of PDR and vitreous hemorrhage in the right eye was examined. BCVA in this eye was 20/100. Fundus examination disclosed severe fibrovascular proliferation around the optic nerve head (Figure 2A). Similarly to Case 1, neovascular vessels were clearly observed in the fibrovascular membrane with the retro-mode imaging function of the F10 SLO (Figure 2B). Two days after $1.25 \mathrm{mg}$ of IVB, fundus examination disclosed regression of
Before IVB

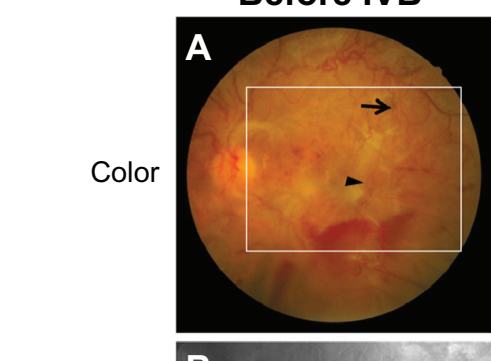

Retro-mode

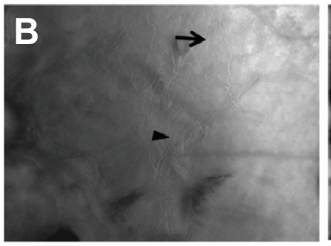

3 days after IVB

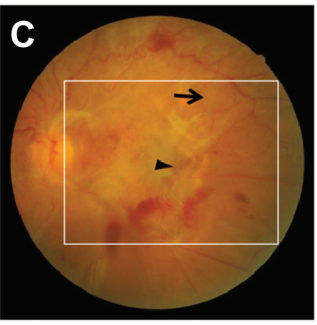

D.

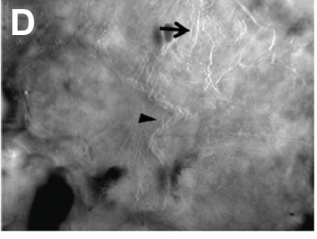

Figure I Case I. A 52-year-old man with very severe fibrovascular proliferation throughout the posterior pole (A). In the retro-mode image, neovascular vessels in the fibrovascular membrane were clearly observed (B). Three days after intravitreal bevacizumab (IVB), most new vessels had regressed in the significantly contracted fibrovascular membrane (C). Neovascular vessel structures were still clearly observed in the retro-mode image after IVB (D). Arrows and arrowheads indicate the same neovascular vessels across images.

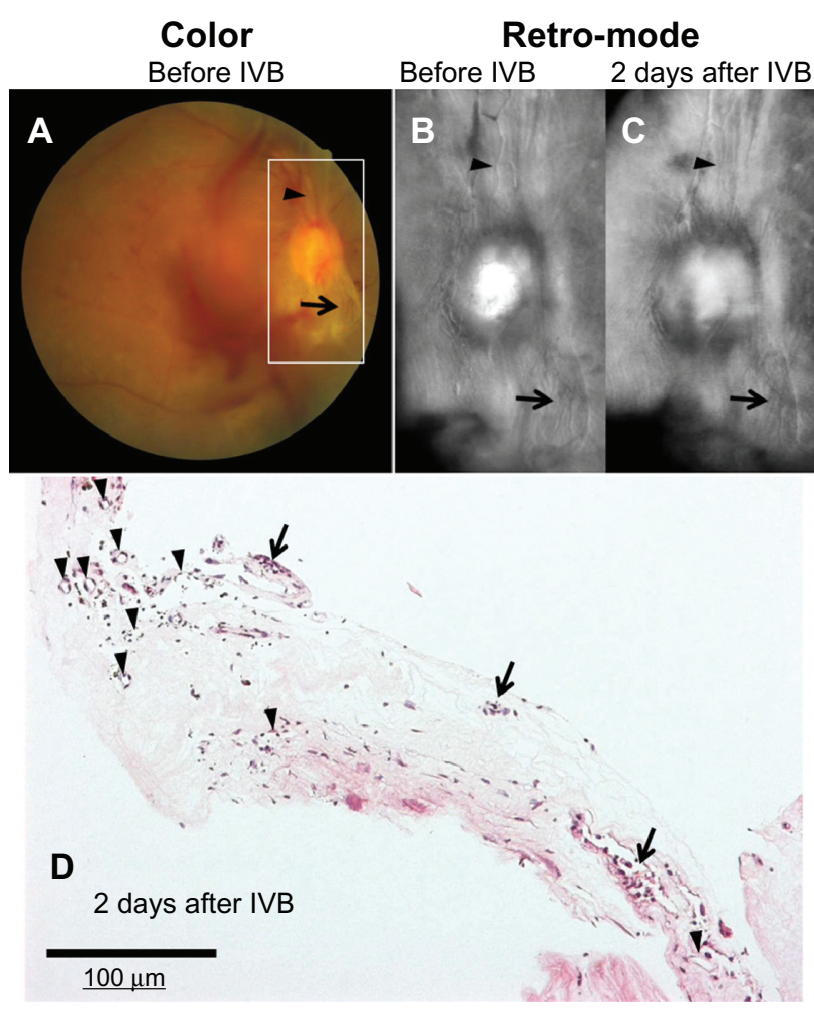

Figure 2 Case 2. A 39-year-old man with severe fibrovascular proliferation around the optic nerve head (A). Neovascular vessels in the fibrovascular membrane were clearly observed in the retro-mode image (B). Two days after intravitreal bevacizumab (IVB), most of the new vessels had regressed. However, in the retromode imaging, neovascular vessel structures were still observed $(\mathbf{C})$. Arrows and arrowheads indicate same neovascular vessels in the different images. Staining of the neovascular tissue from this case with hematoxylin-eosin disclosed that many neovascular capillaries were still present (arrowheads), but that only large vessels contained red blood cells (arrows) (D).

most of the new vessels. However, in the retro-mode image, neovascular vessels were still observed in the fibrovascular membrane (Figure 2C).

To determine the morphological changes in structure of the neovascular vessels after IVB, we prepared formalinfixed paraffin embedded sections from surgically excised fibrovascular membrane 2 days after IVB in this case. Staining of the neovascular tissue with hematoxylin-eosin disclosed that many neovascular capillaries were still present, but that only large vessels contained red blood cells (Figure 2D).

\section{Discussion}

Using the retro-mode imaging function of the F10-SLO, we observed regression of most new vessels in the significantly contracted fibrovascular membrane 2 or 3 days after IVB. Recent reports have described the application of IVB in treating ocular neovascular disorders 
including PDR. ${ }^{8-10}$ IVB has also been reported to be effective in causing the regression of new vessels in PDR. ${ }^{1-3}$ Adjunctive use of intravitreal bevacizumab for severe PDR before vitrectomy significantly decreased bleeding from the vessels of the fibrovascular membrane during the vitrectomy. ${ }^{6,7}$ In case 2 of our study, vitreous surgery was successfully performed and fibrovascular membrane was excised for formalin-fixed paraffin embedded sections 2 days after IVB. These observations suggest that our methods for IVB were appropriate and that 2 or 3 days might be long enough for neovascular vessels to regress, since Arevalo et al ${ }^{11}$ reported that $5.2 \%$ of PDR cases with IVB developed tractional retinal detachment 3 to 30 days after IVB.

We investigated noninvasive evaluation of PDR pathologies before and after IVB using the new indirect viewing system of the retro-mode function of the F10 SLO, and compared the images histologically with surgically excised fibrovascular membrane from one case. In previous reports, ${ }^{4}$ distribution of the cystoid spaces was clearly shown on topographic imaging in diabetic macular edema with the retro-mode imaging function of the F10 SLO. Optical coherence tomography has been especially useful in evaluating the depth distribution of cystoid spaces. However, the horizontal distribution was more easily assessed by retro-mode imaging, suggesting that the two technologies may be complementary. ${ }^{4}$ In our cases of PDR, neovascular vessels in fibrovascular membrane were clearly seen with the retro-mode, even after IVB and without blood flow. Accordingly, the F10 SLO may be useful in evaluating neovascular vessels in fibrovascular membrane in PDR and for determining the precise retinal changes in diabetic retinopathy. In previous reports, neovascular vessels without blood flow ${ }^{1,3}$ and apoptotic cells on the regressing vascular walls ${ }^{2}$ were observed histologically after IVB. In our study, staining of the neovascular tissue with hematoxylin-eosin disclosed that many neovascular capillaries were still present, but that only large vessels contained red blood cells (shown in Figure 2). Our findings of neovascular vessel structures after IVB may be the same as these previously reported regressing neovascular vessels without blood flow.

Although our study suggests that the F10 SLO is useful in evaluating regressing neovascular vessels, there are several limitations to this study. First, our small sample size was small: only two cases were examined, and only one underwent histological examination. Second, the F10 SLO could not detect vascular permeability such as the vascular leakage in retinal and neovascular vessels usually observed in active PDR when examined with fluorescein angiography. In PDR with dense media opacity such as that caused by vitreous hemorrhage, F10 SLO could not be used for fundus examination. The resolution of F10 SLO is not high enough to visualize capillary vessels. These technical limitations of the F10 SLO indicate that it may be most useful in conjunction with other imaging techniques.

In conclusion, we determined the usefulness of the new indirect viewing system of the retro-mode function of the F10 SLO for noninvasive evaluation of PDR pathologies before and after IVB. We demonstrated that the F10 SLO clearly demonstrated the neovascular vessels. Use of an SLO, such as the F10, may provide a unique detailed observation of retinal diseases such as PDR.

\section{Acknowledgments}

This work was supported by a Grant-in-Aid for Scientific Research from the Ministry of Education, Culture, Sports, Science and Technology (No. 21592234, KS, TK), by the Japan National Society for the Prevention of Blindness (KS), and by the Takeda Science Foundation (KS). The sponsors had no role in the study design, the collection analysis, or interpretation of data, writing the report, or the decision to submit the report for publication.

\section{Disclosure}

The authors declare no conflicts of interest.

\section{References}

1. Kubota T, Morita H, Tou N, et al. Histology of fibrovascular membranes of proliferative diabetic retinopathy after intravitreal injection of bevacizumab. Retina. 2010;30(3):468-472.

2. Kohno R, Hata Y, Mochizuki Y, et al. Histopathology of neovascular tissue from eyes with proliferative diabetic retinopathy after intravitreal bevacizumab injection. Am J Ophthalmol. 2010;150(2): 223-229 e1.

3. Pattwell DM, Stappler T, Sheridan C, et al. Fibrous membranes in diabetic retinopathy and bevacizumab. Retina. 2010;30(7): 1012-1016.

4. Yamamoto M, Mizukami S, Tsujikawa A, Miyoshi N, Yoshimura N. Visualization of cystoid macular oedema using a scanning laser ophthalmoscope in the retro-mode. Clin Experiment Ophthalmol. 2010;38(1): 27-36.

5. Tanaka Y, Shimada N, Ohno-Matsui K, et al. Retromode retinal imaging of macular retinoschisis in highly myopic eyes. Am J Ophthalmol. 2010; 149(4):635-640 e1

6. Chen E, Park $\mathrm{CH}$. Use of intravitreal bevacizumab as a preoperative adjunct for tractional retinal detachment repair in severe proliferative diabetic retinopathy. Retina. 2006;26(6):699-700.

7 Ishikawa K, Honda S, Tsukahara Y, Negi A. Preferable use of intravitreal bevacizumab as a pretreatment of vitrectomy for severe proliferative diabetic retinopathy. Eye (Lond). 2009;23(1):108-111. 
8. Avery RL, Pearlman J, Pieramici DJ, et al. Intravitreal bevacizumab (Avastin) in the treatment of proliferative diabetic retinopathy. Ophthalmology. 2006;113(10):1695. e1-e15.

9. Mason JO 3rd, Nixon PA, White MF. Intravitreal injection of bevacizumab (Avastin) as adjunctive treatment of proliferative diabetic retinopathy. Am J Ophthalmol. 2006;142(4):685-688.
10. Spaide RF, Fisher YL. Intravitreal bevacizumab (Avastin) treatment of proliferative diabetic retinopathy complicated by vitreous hemorrhage. Retina. 2006;26(3):275-278.

11. Arevalo JF, Maia M, Flynn HW Jr, et al. Tractional retinal detachment following intravitreal bevacizumab (Avastin) in patients with severe proliferative diabetic retinopathy. Br J Ophthalmol. 2008;92(2):213-216.

\section{Publish your work in this journal}

Clinical Ophthalmology is an international, peer-reviewed journal covering all subspecialties within ophthalmology. Key topics include: Optometry; Visual science; Pharmacology and drug therapy in eye diseases; Basic Sciences; Primary and Secondary eye care; Patient Safety and Quality of Care Improvements. This journal is indexed on

\section{Dovepress}

PubMed Central and CAS, and is the official journal of The Society of Clinical Ophthalmology (SCO). The manuscript management system is completely online and includes a very quick and fair peer-review system, which is all easy to use. Visit http://www.dovepress.com/ testimonials.php to read real quotes from published authors. 